\title{
Contre l'abject : le détective chez Pan Bouyoucas
}

\section{Kathleen Kellett, Université Ryerson}

En 1963, Pan Bouyoucas a quitté le Liban avec ses parents d'origine grecque pour s'établir à Montréal. Depuis la publication de son premier roman Le dernier souffle (1975), il se révèle comme un auteur très prolifique. Peu étudié au Québec, il se trouve souvent relégué à la rubrique de la littérature migrante, étiquette qu'il rejette comme excessivement étroite (voir l'entretien avec Bouyoucas dans ce numéro). Son œuvre, qui se compose de romans, de récits, de contes et de pièces de théâtre, se caractérise par la polyvalence. Le roman policier joue un rôle privilégié dans l'œuvre de Bouyoucas,; selon lui, « Tout comme la tragédie, le polar donne une image plus vraie de l'humanité, dans le sens qu'il révèle les forces obscures tapies dans nos entrailles, notre capacité à la violence, car nous sommes tous capables d'un tel geste » («Entretien avec Pan Bouyoucas », $15)$.

Souvent méprisé pour son association avec le cliché et le pré-fabriqué, le polar partage des traits importants avec les genres littéraires les plus prestigieux. Le suspense essentiel au polar, son élan téléologique, se retrouvent à des degrés différents dans toute fiction. Le meurtre qui lance si souvent l'intrigue policière, est un ressort dramatique qui déclenche l'enquête destinée à l'élucidation du mystère. Le crime, surtout le meurtre, instaure une rupture dans l'ordre social, signalant la présence de l'abject, comme l'affirme Julia Kristeva dans Pouvoirs de l'horreur: «Tout crime, parce qu'il signale la fragilité de la loi, est abject, mais le crime prémédité, le meurtre sournois, la vengeance hypocrite le sont plus encore parce qu'ils redoublent cette exhibition de la fragilité légale » (12). Prenant racine au moment où l'individu commence à se rendre compte de la séparation entre le soi et le corps maternel (20), l'abject se situe à la frontière entre le soi et l'Autre, entre l'ordre et le désordre social, la raison et la folie, la vie et la mort. La pourriture, le sang renversé, la merde évoquent tous l'abject car ils mettent en question l'intégrité du corps comme entité étanche. Le cadavre en particulier provoque le sentiment de l'abject : «Le cadavre - vu sans Dieu et hors de la science - est le comble de l'abjection. Il est la mort infestant la vie. Abject» (11-12). Confiner l'abject par la sublimation est possible selon Kristeva mais l'abject menace à tout moment de ressurgir. La clôture typique de l'intrigue policière - la suppression juridique de la menace sociale que représente la criminalité - peut être vue comme une forme de 
sublimation.

Outre cette fascination avec l'abject, ce que le polar a de particulièrement caractéristique est le personnage canonique du détective. À la fois représentant de la loi et fin limier, le détective a le devoir de restaurer l'ordre social déchiré par l'acte de violence. Lorsque le détective est policier, il bénéficie des outils de l'institution policière et, en principe, se met au service de la loi imposée par l'État. Je me penche ici sur deux romans de Bouyoucas, La vengeance d'un père (1997) et Cocorico (2011), en considérant le polar comme lieu privilégié de la manifestation de l'abject où le rôle principal du détective est de mener à bien son enquête de sorte à mater, circonscrire et sublimer l'abject. Toujours est-il que les textes de Bouyoucas résistent à la pulsion téléologique du polar pour véhiculer une vision plus nuancée de la condition humaine.

Comme l'indique Charles Brownson dans The Figure of the Detective, en tant que genre canonique, le polar a tendance à favoriser une intrigue qui aboutit à un dénouement bien défini (83). En même temps, le rétablissement de l'ordre social favorisé par l'institution judiciaire dans le polar classique privilégie le statu quo plutôt que la contestation sociale ou politique, comme le remarque Kathleen Klein en examinant le polar féministe (200). Or, tout en valorisant le rôle du détective, Bouyoucas jette un regard critique sur la société, y apportant une perspective défamiliarisante qui problématise le rôle de l'Autre. Le détective sous sa forme idéale paraît comme représentant de la raison et de l'ordre social, un rempart contre l'abject et le chaos ; pourtant, l'abject se défend et se défoule tout comme la trame narrative résiste à toute résolution facile.

\section{Le détective contre l'abject}

L'imaginaire social occidental est peuplé de détectives canoniques dont, par exemple, Sherlock Holmes d'Arthur Conan Doyle, Jules Maigret de Georges Simenon et, au Québec, Maud Graham de Chrystine Brouillet. Brownson insiste sur les origines du polar au dix-neuvième siècle en Grande Bretagne et en France où l'esprit rationaliste émerge comme axiologie dominante (11). Issu des forces de sécurité établies par Robert Peel en Angleterre dans les années 1830 ainsi que de la Sécurité nationale établie en France à l'époque de Napoléon, les forces policières mettent en vedette le détective. Il est à la fois collectionneur et lecteur d'indices; il tire des conclusions de son enquête afin de trouver les responsables d'un crime. Sa réussite à élucider les crimes sert à rassurer le lecteur sur le bien-fondé de l'esprit scientifique. Pour Nicolas Xanthos, c'est l'étape de « la sémiotisation 
narrative » (360) qui constitue le travail essentiel du détective : Comment lire les indices ? Entre plusieurs interprétations possibles, comme en reconstruire la bonne ? Dans le polar canonique, la logique doit prévaloir face aux forces du désordre social. Le détective paraît un peu comme le preux chevalier, destiné à protéger la société, à témoigner de la corruption de la ville sans en être touché car il s'agit d'un genre éminemment urbain (voir Highmore 98). Dans ces deux romans de Bouyoucas, la capacité du détective de reconstituer le récit qui correspond le mieux aux faits, dépend de sa fidélité aux méthodes empiriques, ses dons d'abduction et son refus de se laisser influencer par les préjugés de la société qui l'entoure. Dans le roman policier, le détective est donc représentant de l'esprit rationaliste et défenseur du bien-être de la société. Évidemment, nous tombons dans le cliché mais c'est justement un des plaisirs du polar dont les structures et les motifs sont tellement prévisibles que l'auteur et le lecteur peuvent partager le plaisir de jouer à la subversion du genre et de ses éléments consacrés.

La vengeance d'un père et Cocorico traitent des motifs du polar de façons très différentes. Dans La vengeance d'un père, il s'agit d'un roman policier qui commence par un acte de violence, la défiguration d'une jeune femme Eléna Fokas, fille d'un immigrant grec à Montréal, ce qui entraîne le suicide de celle-ci ainsi que le meurtre de Marc Saint-Aubin, fils d'un ministre souverainiste qui essaie d'entraver l'enquête de Georges Soucy afin de protéger son fils. Il est à noter que dans la version originale du roman paru en 1997, le père s'appelle Réal Lachapelle et le fils, Marc Lachapelle. L'étude onomastique pourrait souligner le renvoi ici au contexte religieux du Québec d'antan avec « Lachapelle » alors que «Saint-Aubin » suggère la puissance sociale, une nouvelle aristocratie. En dépit de la corruption et des luttes intestines dans sa ville, le sergentdétective Soucy doit examiner les faits, interroger les suspects et éviter les fausses pistes pour élucider les crimes qui sèment la panique dans la communauté montréalaise.

Dans Cocorico, il ne s'agit ni d'attentat ni de meurtre ; plutôt le détective canonique du polar y représente le raisonnement et le sacrifice personnel qui s'opposent à la déraison et à l'isolement social typique de celui qui est atteint de la maladie d'Alzheimer, syndrome qui mène inévitablement à l'abjection, brouillant les frontières entre la raison et la déraison. Le sergentdétective Vass Levonian est la création de l'auteur Léo Basilius qui, sur le tard de la vie, décide de se consacrer à un genre d'écriture plus prestigieux et plus optimiste que le polar. Dans son dernier polar, le seizième de la série, le détective git dans le coma entre la vie et la mort, ayant été blessé au cours d'une enquête. Au grand désarroi du personnage, son auteur n'a aucune intention 
de le ressusciter et un grand débat s'ensuit entre le créateur et sa création. En examinant les deux romans de Bouyoucas, il est évident que le rôle idéal du détective est axé sur le sacrifice et la responsabilité envers l'Autre, principes qui l'amènent à vouloir maintenir l'ordre social. Abandonné de son auteur, Levonian se doit d'aider son créateur à lutter contre la démence progressive. Pour sa part, Georges Soucy se bat contre les forces du désordre social qui risquent de l'engloutir dans cette société divisée du Montréal post-référendaire.

\section{La vengeance d'un père : politique et patriarcat}

Dès le début de La vengeance d'un père, le détective Soucy établit son rôle comme défenseur intègre de l'ordre social en faisant une promesse solennelle de trouver l'agresseur qui a défiguré Eléna Fokas. Il exige du père de la victime une promesse en retour : «Qui que ce soit, nous ferons tout pour le trouver, je vous en donne ma parole. [...] Je comprends votre douleur, monsieur Fokas. Mais vous ne ferez pas justice vous-même. J'ai votre parole ? » (49-50) En tant que père lui-même, il ressent de la compassion pour Yannis Fokas ; il s'engage à faire de son mieux pour réparer les dommages infligés à la famille. Soucy convient parfaitement au stéréotype du policier qui a consacré sa vie à son travail et dont la vie familiale a gravement souffert en conséquence. Son ex-femme est alcoolique ; sa fille Dominique est souvent distante de lui. Il fait un rêve récurrent où il est en vacances à Virginia Beach aux États-Unis avec son ex-femme et sa fille, où c'est toujours lui qui doit s'occuper des préparatifs du voyage sans jamais avoir l'occasion d'aller se baigner dans l'océan lui-même. Alors que les deux autres sont à la plage, dans son rêve, il n'en bénéficie jamais. Il s'agit d'un homme intègre et altruiste mais en même temps vulnérable, ce qui brouille sa perception des faits devant lui.

Le devoir professionnel de Soucy envers la famille Fokas s'accroît lorsqu'Éléna se suicide en se jetant sous les rails du métro, profondément déprimée par cette défiguration qui aurait mis fin à ses rêves d'actrice professionnelle. Suspect dans l'agression contre Éléna, Marc Saint-Aubin, voleur et vendeur de drogue mais aussi fils d'un politicien souverainiste réputé, est poignardé sur les lieux mêmes où Éléna a été attaquée. Ces deux morts perturbent l'ordre social et c'est à Soucy d'en éclaircir les circonstances, de découvrir les coupables et de les amener à la justice. Malheureusement, le détective est entraîné dans le tourbillon grandissant du monde abject d'une société montréalaise perturbée par des luttes intestines axées sur l'identité ethnique et la classe sociale. Au cours du roman, Soucy doit essayer de peser la valeur de trois pistes d'interprétation 
concurrentielles : une lecture politique en fonction des tensions sociales dans la communauté montréalaise ; une lecture judiciaire en fonction des activités criminelles associées à cette communauté de jeunes gens ; une lecture psychologique qui tient compte des conflits œdipiens.

La lecture politique est favorisée par les membres de la communauté montréalaise. Tout ce drame se passe dans le contexte du Québec post-référendaire évoqué dès le début du roman lorsque Yannis Fokas réfléchit à l'impact du « référendum sur l'indépendance du Québec, où l'on avait accusé les minorités ethniques de la défaite du Oui » (10). Ici, il fait allusion aux commentaires de Jacques Parizeau, chef du Parti québécois, après le référendum de 1995 : «C'est vrai, c'est vrai qu'on a été battus, au fond, par quoi ? Par l'argent puis des votes ethniques, essentiellement » (Archives de Radio-Canada, 30 octobre 1995). Avant la nouvelle de l'attentat contre sa fille, Yannis Fokas n'est pas vraiment troublé par l'impact du référendum : «Estime-toi heureux de vivre dans un pays où l'on ne sort pas les couteaux lorsqu'on perd les élections », dit-il à son beaufrère (10). Cependant, au cours de l'enquête, la méfiance mutuelle entre immigrants et Québécois de souche commence à influencer sa façon de penser. En tant que détective, Soucy a l'obligation de restaurer l'ordre dans sa ville sans prêter attention aux voix de la foule. Cependant, ses propres allégeances politiques et familiales l'empêchent de voir clair alors que les médias locaux brouillent les pistes.

$\mathrm{Au}$ fond, les indices sont minimes : l'empreinte des bottes Kodiak ; l'anorak rouge de l'agresseur ; le fiancé jaloux qui a quitté le bar Marechiaro bien avant l'agression ; un suspect au bar, à savoir, Marc Saint-Aubin, qui aurait crié des paroles menaçantes en réponse à une réplique critique à propos du référendum : «Tu achèves ton temps, ma noire ! » (88). Le seul témoin de l'agression est Anna, la sœur de la victime, qui a été assommée ce soir-là elle aussi. Les médias et les groupes communautaires se font concurrence pour offrir leur propre version des événements : Penny O’Neill (anglophone fédéraliste), du journal The Gazette ; Michael Léontis (communiste grec), président de l'Association des parents grecs ; Nestor Petropoulos (fédéraliste), de la station radiophonique de langue grecque CHRM ; Jacques Murphy (francophone souverainiste), du Journal de Montréal. Les noms constituent des indices de la diversité de la société québécoise ; les noms de famille irlandais O'Neill et Murphy rappellent le rôle que le Québec a joué au dixneuvième siècle en accueillant ceux qui fuyaient la famine en Irlande alors que les noms grecs racontent l'histoire d'une immigration plus récente. Dans l'espace urbain du polar - ici la ville de Montréal dans toute sa diversité - la foule élève la voix, révélant surtout un certain taux 
d'intolérance. L'auteur nous plonge dans le trope de la ville corrompue, lieu privilégié du roman noir : «Monde de la peur, de la solitude, de la puissance, de l'orgueil, du danger, de la misère, de la richesse, de la réussite, des bas-fonds, telle est la ville du polar » (Blanc 33). Dans un entretien, Bouyoucas fait l'éloge des bienfaits potentiels du dialogue dans l'espace urbain : «...pour moi, la Cité est un endroit offrant les plus grandes possibilités de dialogue. Et à travers l'histoire, le symptôme le plus révélateur de l'échec urbain a souvent été l'absence de dialogue. Pas nécessairement un silence, mais le murmure complaisant d'un chœur de citoyens dévidant les mêmes inanités » («D'autres rêves », 108). Dérouté par les voix tumultueuses des membres de la Cité, hanté par le souvenir des policiers qui ont assassiné impunément son père en Grèce, Fokas choisit de poursuivre lui-même l'individu qu'il identifie à tort comme responsable de l'agression contre sa fille. Fokas se convainc que les lacérations sur les joues de sa fille sont censées représenter le chiffre 101, rappel de la loi 101instaurée en 1977 par le Parti québécois : «Était-ce l'intention de l'agresseur? se demanda-t-il. De se venger des propos antinationalistes de sa fille en gravant sur son visage le numéro de la loi qui avait fait du français la langue officielle du Québec ? » (153). Il ne lui en faut pas plus pour passer à l'acte d'agression lui-même : « Cette preuve lui permit de justifier à ses yeux le crime qu'il se devait de commettre » (186). Faisant fi de l'avertissement de Soucy, il finit par poignarder Saint-Aubin, convaincu par sa propre lecture politisée de l'attentat contre sa fille.

Pour sa part, Soucy commence par considérer les suspects dans le cercle de jeunes gens autour de Saint-Aubin. Exaspéré par la lenteur de l'enquête ainsi que par la pression politique, Soucy déroge de son devoir de policier en utilisant des techniques de pression non-autorisées pour interroger une jeune prostituée qui fait partie du gang de Saint-Aubin. Plus tard, démis pour un temps indéfini de ses fonctions professionnelles pour sa conduite indigne, Soucy n'est pas en mesure de révéler à son supérieur la conviction que c'est en fait Fokas qui a poignardé SaintAubin. Lors des funérailles d'Éléna, il guette les réactions du père en cherchant des indices de culpabilité. Bien que le crime de Fokas ne soit jamais puni par l'institution judiciaire, les événements bouleversent la psyché du père qui vit désormais au bord de la folie, atteint de l'abject entraîné par le fardeau du deuil et de la culpabilité.

Afin de résoudre le crime, il faudrait au détective plutôt une compétence en dynamique familiale et peut-être en mythologie grecque, car le nœud de tous les crimes dans le roman est la perturbation de la structure familiale patriarcale, comme le suggèrent les paroles de Harry Baltas, 
le père du fiancé d'Éléna : «Vous avez de la chance d'avoir une fille, avait dit Harry Baltas. Vous connaissez 1'histoire d'Edipe... » (173). À son insu, il touche au cœur du mystère. Éléna a été défigurée par Julien Beaulieu, fils illégitime qui a voulu se venger contre Baltas, son père biologique, en s'en prenant à la fiancée du fils légitime Tony Baltas. On remarque le caractère misogyne du crime : on s'attaque à l'homme puissant en détruisant la beauté de la fille qui accompagne son fils. La femme-victime n'est qu'un instrument de vengeance pour Beaulieu. Le fils illégitime punit le père en s'attaquant à la femme qui « appartient » au fils légitime rival. Par la suite, le meurtre de Saint-Aubin par Fokas résulte d'une mauvaise lecture des indices, dictée par la colère, la culpabilité, le malentendu culturel et le désir de Fokas de se venger du meurtre de son propre père en Grèce aux mains des agents de police corrompus.

Le détective Soucy est aveuglé par sa propre participation aux querelles politiques qui affligent sa ville natale. La xénophobie envahit sa pensée : «Belle société en perspective ! Les Juifs qui parlent des Québécois, des Noirs et des latinos en se bouchant le nez. Les Grecs qui accusent tous les autres de conspirer contre eux, alors qu'ils volent en catimini nos pères et nos filles » (270). Comme l'indique Robert Mane, Soucy, comme les autres personnages montréalais ici, souffre d'un profond « mal être »: «Le mal est dans leur incapacité foncière à communiquer » (44). Soucy ne se rend pas compte des dangers qui menacent sa fille Dominique qui, elle aussi, sort avec un des fils de Baltas. C'est au moment où il cède à sa colère contre sa fille en jouant le rôle de père autoritaire que sa fille court le plus grand risque. Le téléphone sonne mais il refuse de répondre : «Qui m’appellerait de là ? Dominique ? » Il hésita un instant devant l'appareil, un instant qui allait revenir le hanter pour le reste de sa vie. «Non, je ne réponds pas. Qu'elle souffre ! (295). Ce refus rappelle celui de Fokas la nuit où Éléna lui a téléphoné pour lui souhaiter «Bonne année », la nuit où celui-ci a choisi de ne pas répondre pour punir sa fille de n'être pas restée avec la famille : «Cependant, quand ses filles téléphonèrent à minuit pour souhaiter à leurs parents la bonne année, il refusa de leur parler. Pour être logique. Car il était très important à ses yeux qu'un homme fût conséquent »(11). Ce désir de contrôler et de manipuler leurs filles empêchent les pères d'agir de façon conséquente avec leur amour paternel, ce qui entraîne la catastrophe. Beaulieu essaie d'écraser Anna Fokas et Dominique Soucy avec sa voiture et celle-ci arrive à l'hôpital gravement blessée. À cause de son égoïsme, Soucy n'est pas en mesure de bénéficier des indices recueillis par sa fille et par Anna qui ont repéré avant lui le danger que constituait Beaulieu. 
En identifiant enfin Beaulieu comme l'agresseur d'Éléna Fokas et de sa propre fille, Soucy doit reconnaître la primauté de la dynamique familiale dans la version véridique des événements. Des indices négligés auparavant à cause d'une mauvaise lecture des événements acquièrent un nouveau sens : une référence par le ministre péquiste Jacques Saint-Aubin à une affaire de harcèlement sexuel de la part de Baltas père ; les plaintes de son propre père Maurice Soucy au sujet de son ancien patron Harry Baltas qui lui aurait «volé » une Gaspésienne du nom de Thérèse Beaulieu (261). Pris par sa haine de l'Autre, le détective Soucy n'a pu accomplir son devoir de policier. Ni la famille Fokas ni la société ne se trouve hors danger puisque Beaulieu reste toujours en liberté - infraction flagrante aux conventions du roman policier canonique qui favorise inévitablement la clôture et le rétablissement de l'ordre social. Bouyoucas choisit d'identifier le malfaiteur sans offrir au lecteur la rassurance des sanctions sociales qui seraient plutôt habituelles dans le polar. La ville reste tout aussi corrompue qu'avant - remplie de criminels dangereux comme Beaulieu et de gros hommes d'affaires louches comme Baltas et Saint-Aubin pères. Le détective n'échappe pas à la corruption malgré ses bonnes intentions du début. Lorsque le lieutenant Bastien retire Soucy de cette enquête, c'est le signal d'un retour possible à un idéal d'intégrité. C'est Bastien qui prononcera la condamnation officielle de Baltas et des hommes puissants de son genre : «'À force d'habiter l'étage le plus élevé de Montréal ou parce que la chance lui a toujours souri de façon insolente, il a fini par se croire infaillible', notera-t-il plus tard dans son rapport, dans une de ses rares envolées philosophiques » (301). Il se contente d'avertir Baltas du danger qui guette les petites amies de ses fils légitimes tant que Beaulieu restera en liberté. L'ouverture du récit met en question la possibilité de restaurer l'harmonie sociale dans une ville où la xénophobie est à fleur de peau. Comme le constate Maïr Verthuy, Bouyoucas montre bien que la xénophobie menace toute la communauté montréalaise : « Il nous rappelle néanmoins non seulement que les immigrés portent une part de responsabilité dans cette situation mais aussi que la société d'accueil souffre également de son incapacité de se renouveler en acceptant l'apport des nouveaux venus » (181). En tant que détective, Soucy n'échappe pas à la corruption ; plutôt il se fait engloutir dans l'abject, la corruption, l'égoïsme, la haine de l'Autre. Pourtant, l'idéal de la rationalité et de la justice sociale persiste, incarné par le lieutenant Bastien. À la fin du roman, l'aperçu d'un nouvel ordre social est surtout signalé par la résilience d'Anna Fokas. Elle est fermement intégrée à la société québécoise, ouverte d'esprit, imbue de l'agentivité de la femme qui a dépassé le patriarcat. Elle demande à sa grand-mère comment elle parvient à «aller toujours 
de l'avant », ce à quoi la vieille dame répond : «Pourquoi ? Peut-on aller de l'arrière ?» (308309). Tandis que son père sombre dans la folie, Anna poursuivra son chemin de Québécoise émancipée sans oublier ses racines grecques.

Malgré tous les sacrifices impliqués dans le travail policier, le détective n'arrive pas à éliminer ni les manigances des puissants ni la violence qui menace les vulnérables. L'abject risque de surgir à tout moment. Pourtant, l'idéal de la rationalité et l'opposition active à l'abject persistent chez les policiers intègres comme Bastien ; le refus de la xénophobie et du système patriarcal persistent chez les femmes pragmatiques et agissantes comme Anna Fokas. Ce sont d'ailleurs ces valeurs de rationalité, d'ouverture d'esprit et d'intégrité qui soutiennent l'axiologie du roman Cocorico.

\section{Cocorico : la raison, la déraison et l'abject}

Le roman Cocorico explore l'angoisse suscitée par l'appréhension de la perte des facultés intellectuelles. Le pouvoir de la logique constitue une bouée de sauvetage qui apporte de l'espoir à l'écrivain Leo Basilius, tiraillé entre la lucidité et la démence, aux bords donc de l'abject, ce territoire flou où les frontières entre la vie et la mort commencent à s'effacer. L'auteur voudra s'éloigner du genre méprisé du polar pour se faire une réputation d'auteur sérieux, voire illustre, créateur d'une œuvre profonde et digne. Pour cela, il revient à l'île de Nysa en Grèce où il a écrit ses œuvres de jeunesse, des nouvelles et quelques poèmes. À la fin du seizième polar de Basilius, Levonian est suspendu entre la vie et la mort, au bord de l'abject, gravement blessé au cours d'une enquête où il protégeait une petite fille contre les prédations d'un pédophile sadique. Une longue carrière de détective, vedette de roman et de films populaires, s'achève. Un des ressorts dramatiques du récit est donc l'attentat mystérieux de la part de l'auteur contre son propre personnage pourtant fort rentable. Levonian emploie toutes les remontrances et tous les arguments possibles pour convaincre son auteur de le ramener à la vie active. Il met ses dons de raisonnement au service de son auteur, en espérant la clémence. D’une part, le récit offre une lecture réaliste de la vie d'un homme de plus en plus accablé par la perte progressive de la raison ; d'autre part, on y trouve une lecture méta-discursive axée sur les motifs du polar valorisant les méthodes empiriques, l'argumentation et l'altruisme qui se rattachent au personnage du détective.

À première vue, le polar paraît dans Cocorico comme un mauvais genre, imbu de violence et de désespoir. C'est justement cette perspective fataliste envers la brutalité de l'existence que 
condamne Basilius, «le maître incontestable du polar canadien » (8), et qui l'amène à vouloir abandonner ce genre paralittéraire. Il commençait à croire que les polars « ... donnaient l'impression que le vice régnait à l'état endémique en Amérique du Nord, que les villes étaient pourries par la drogue et le crime, que dans chaque quartier les meurtres étaient aussi fréquents que les vols à l'étalage » (32). On voit une condamnation encore plus méprisante du genre chez un des personnages secondaires, amateur de la littérature plutôt classique :

$\mathrm{Ne}$ le prenez pas personnellement, dit-il au roi du crime, je n'aime pas les polars. Les personnages sont trop stéréotypés, les histoires, trop prévisibles. Mais ce que je trouve le plus rebutant, c'est leur prétention d'explorer les tréfonds de l'âme humaine alors qu'ils ne font qu'exploiter ses aspects les plus pervers et violents, avec quelques boniments sociopolitiques pour se donner bonne conscience. (21)

Basilius a démarré sa carrière d'écrivain de bestsellers en puisant dans son expérience de rédacteur d'un bulletin mensuel du syndicat des policiers, boulot qu'il avait accepté en bon père de famille pour subvenir aux besoins de sa femme et de ses enfants. Ce roman populaire, intitulé Janus \& associés, « vendu à plus de cent mille exemplaires » et «traduit en une dizaine de langues » (16), avait néanmoins un message social important puisque Basilius l'a basé sur le cas véridique de l'incarcération injuste d'un jeune autochtone canadien. Si Levonian lui-même rappelle à son auteur que «Le monde, le vrai, n’est qu'un gros merdier, Leo, ici comme ailleurs » (53), le détective représente toujours ici l'espoir que la raison et le sacrifice personnel peuvent l'emporter sur la folie et l'égoïsme.

Autour de Basilius, personne ne s'aperçoit du lien entre son refus de reprendre le polar, genre méprisé, et la peur de la démence. Écrire pour la postérité, c'est écrire contre la mort, but égoïste que sa femme Muriel n'apprécie pas : «... tu penses que ta vie aura été une faillite si ton plan d'immortalité ne réussit pas. Comme si moi, les trois enfants que je t'ai donnés, et leurs enfants à eux, ne comptions pas à tes yeux autant qu'une page du chef-d'œuvre qui est supposé assurer ta postérité » (79). Malgré sa connaissance profonde de son mari, elle interprète mal les indices de l'affaiblissement mental de Basilius. Elle ne voit dans son manque d'intérêt pour la vie sentimentale tourmentée de leur fille et sa façon de murmurer le nom de Loraine dans son sommeil que la distraction égoïste d'un écrivain obsédé par son travail. Elle croit reconnaître une dynamique familiale bien connue d'elle - celle qui reflète l'image du père écrivain, gagne-pain, et de la femme consacrée à l'établissement de l'harmonie domestique - mais cette lecture des indices se révèle comme fausse piste. 
C'est le détective Levonian, le double de Basilius, qui finit par déceler les signes de la démence précoce chez son auteur. S'il s'élève contre la décision de Basilius d'abandonner le polar, c'est parce qu'il tient à lutter contre l'extinction de sa propre vie inextricablement liée à celle de son auteur. Le thème du dédoublement s'annonce dans le titre du premier polar de Basilius, Janus et associés, Janus étant le dieu romain à deux visages qui regarde à la fois vers le passé et l'avenir, celui qui favorise les nouveaux départs et les transitions (Chevalier et Gheerbrant 530). Si l'on considère que le nom « Basilius » est l'équivalent du nom grec « Vasili », on voit bien que l'auteur et son personnage sont liés par des initiales inverties : LB (LV); VL (voir aussi l'entretien avec Bouyoucas). Face à la décision de son auteur de l'abandonner, Levonian insiste à plusieurs reprises sur les sacrifices qu'il a faits en tant que détective. Il reproche amèrement à son auteur d'avoir fait mourir systématiquement l'une après l'autre les femmes dans sa vie ainsi que l'enfant que portait sa dernière femme Véronique : «Pourquoi toutes ces morts et trahisons, Leo, toutes ces lumières perdues, tous ces chagrins ? Les femmes que j'ai aimées. L'enfant que j'attendais. Les amis et collègues que j'ai perdus. Ils n'ont vécu et trépassé que comme ressorts dramatiques, pour corser tes récits et te rendre riche et célèbre ? »(108). Mais Levonian reproche surtout à son auteur de lui avoir ôté son rêve de la vie après la retraite : «Le paradis bleu de la retraite, en Floride ou dans les Caraïbes. Le rêve de chaque policier » (92). Comme Georges Soucy avec son rêve toujours raté de vacances en famille à Virginia Beach, Levonian recherche la récompense de la retraite rêvée : «Depuis seize polars, tu commandes et j'obéis. Est-ce trop demander, à mon tour, une retraite dans un paradis bleu, avec quelques souvenirs d'enfance, une photo de mes parents, une femme qui me donnera un enfant ou deux ? »(117). On remarque que ce que Levonian désire avant tout est ce que possède Basilius - femme, enfants, douceurs de la vie - et tout ce que celui-ci semble mépriser. Plongé dans le coma par son auteur sans pitié, Levonian fait face aux mêmes angoisses existentielles que son créateur : la peur de la mort, de la perte des facultés intellectuelles. Dans ce contexte, le « rêve bleu », l'espoir du paradis, se heurte à l'appréhension du néant.

À Nysa, Basilius cherche l'inspiration qui lui permettrait de créer un roman axé sur la joie et destiné à lui apporter la gloire littéraire. Il croit trouver cette inspiration dans une question posée par une petite fille sur l'île grecque : «Pourquoi le coq crie-t-il à l'aube ?» (67). Avec son alter ego Levonian, il réfléchit à cette question en s'asseyant devant des poulaillers à l'aube. Basilius devient la risée des gens de l'île lorsqu'il se met avec son personnage à débattre cette question de lumière et d'ombre : est-ce que le coq crie de joie en voyant le soleil se lever ou est-ce qu'il crie 
de désespoir parce qu'il se rend compte de nouveau chaque jour qu'il est incapable de voler ? Basilius a beau se rendre compte que Levonian essaie de le distraire en évoquant cette image du coq qui se lamente des effets de la gravité, il est obsédé par ce mystère. Il se lève tôt chaque matin et regarde le coq en essayant de ramasser des indices pour trancher la question. Cette recherche de la raison d'être du coq se rattache directement aux souvenirs que Basilius détient de son propre père atteint de la maladie d'Alzheimer : «Son obsession pour le chant du coq lui paraissait si insensée, à présent, qu'elle lui rappelait celles de son père quand il avait été atteint de la maladie d'Alzheimer » (120). Alors que sa femme Muriel s'exaspère de ses troubles de mémoire, Levonian est au courant de l'angoisse de son double : «Je vais croire que t'as vraiment hérité de l'Alzheimer de ton papa $\gg(71)$.

Levonian illustre surtout le rôle du détective canonique : collectionneur d'indices, logicien méthodique, limier exceptionnel. L'agent de police a accès à cette expertise à l'intérieur de l'institution policière mais il fait confiance aussi à l'abduction, ce saut d'intuition qui puise dans des savoirs inconscients. Pour analyser le chant du coq, Levonian utilise les méthodes que Basilius a déjà attribuées à son personnage en commençant par l'abduction, processus qui fait écho à celui dont l'auteur se sert pour créer le plan de chaque roman : «C'est peut-être la bougie, lui disait son instinct, usant d'une métaphore qu'il avait mise dans un de ses polars dans la bouche de Levonian»: «J'allume la bougie et, à la lueur de sa flamme, je trouve enfin le commutateur. J'éteins la bougie et, avec la lumière qui illumine la pièce dans laquelle je me trouve, je réussis à trouver le commentateur de la pièce suivante, et ainsi de suite, jusqu'à ce que tout le bâtiment soit illuminé » (70; en italique dans le texte). Chaque point de lumière en entraîne un autre jusqu'à ce que tout soit illuminé. Stéphanie Walsh Matthews insiste sur le caractère primordial de cette intuition illuminatrice : «L'abduction, acte de priméité, reflète ce qu'il y a de plus intelligent chez l'enquêteur. Loin d'être la propriété du rationnel, l'abduction réside dans l'intuitif » (282). Cependant, l'intuition n'est pas suffisante en soi. Levonian rappelle à Basilius l'importance de la «règle d'or » de toute investigation : refuser le préconçu, s'appuyer sur l'observation, procéder par la logique inductive : «T'as la jugeote tellement obnubilée par ton thème, poursuivit Levonian en lui emboîtant le pas, tu voudrais tellement que le chant du coq soit un hymne à la vie, que tu arranges et fardes l'évidence pour qu'elle appuie ta théorie, faisant fi de la règle d'or de chaque enquête. Évacuer toute idée préconçue et ne tenir compte que des faits (98)». Les puissants du monde se sont déjà servis du coq comme symbole pour renforcer leur propre autorité sur le peuple : 
«Pas étonnant que certains ont fait du coq un symbole religieux d'espoir et de foi, que d'autres l'ont associé à l'idée de la nation et d'une nouvelle ère, alors que des rois l'incorporaient dans leurs blasons » (94). Pour sa part, Basilius préfère s'appuyer sur l'autorité de la méthode empirique : «Et avant de lui prêter les émotions et les intentions humaines dont les prêtres, les nationalistes et autres charlatans l'ont affublé, je devrais revenir l'observer demain matin pour vérifier s'il regarde chaque aube le lever du soleil avec autant d'émerveillement » (94). Bouyoucas reprend ici un thème récurrent dans son œuvre, celui de la résistance contre les abus commis par les puissants de ce monde, dont, par exemple, Baltas et Saint-Aubin pères dans La vengeance d'un père. Dans ce contexte, la logique inductive basée sur l'observation est un rempart essentiel non seulement contre la démence sur le plan individuel mais aussi contre l'abus de pouvoir sur le plan social.

Levonian continue d'encourager son auteur en insistant sur l'optimisme au fond de chaque être :

Leo, polar après polar, même quand chaque illusion que je nourrissais se révélait amère, je continuais à bûcher et à rêver de mon petit paradis. Même dans le coma, chaque fibre de mon corps rêve d'une glorieuse résurrection où l'océan effacerait les rides de mon cœur et comblerait enfin mes désirs. Parce que tu m'as fait ainsi. Et tu m'as fait ainsi parce que tout créateur programme ses créatures pour espérer coûte que coûte, même face à l'inéluctable. De même pour le coq. Il est animé d'un tel espoir que toutes les limites deviennent des mirages. Et chaque aube, il voit dans le soleil levant la promesse d'un nouveau jour fait d'envols et d'odyssées. (137)

Cependant, cet optimisme s'écroule face aux avances inéluctables de la démence. À la fin du roman, assis devant le poulailler, Basilius ne sait plus quelle direction prendre pour rentrer et fait appel à Levonian, représentant de la raison, qui essaie d'aider son créateur à retrouver le chemin de retour : «Aide-moi, Vass. Sinon Loraine va encore se faire du souci et ne me laissera plus sortir » (140). Perdu dans ses souvenirs de jeunesse, il a oublié jusqu'au nom de sa femme de trente-cinq ans en la confondant avec sa compagne d'aventures en Grèce des années 1970. Finalement au bout de ressources intellectuelles, Levonian et son créateur restent figés par l'indécision, incapables de relever des indices qui pourraient les aider à se situer dans l'espace. L'auteur du polar, «maître de la sémiotisation narrative », n’arrive plus à formuler une séquence narrative qui se tient. Sa création, le détective Levonian, ne retrouve plus sa facilité de raisonnement. Sur le plan personnel, c'est le triomphe de l'abject. Par contre, même si l'on sait que Basilius n'écrira jamais son magnum opus, le pouvoir du récit littéraire persiste. C'est peut- 
être le message que sa femme essayait de lui communiquer en lui faisant écouter les histoires merveilleuses que les habitants de l'île voulaient lui raconter avec l'espoir que l'auteur s'en inspirerait pour un de ses polars.

\section{Le détective, homme de toutes les saisons, rempart contre l'abject}

Chez Bouyoucas, le détective sous sa forme idéale, tel que représenté par Vass Levonian, paraît comme exemplaire de l'homme rationnel voué à la justice et à l'ordre social. Il s'oppose à l'égoïsme ainsi qu'à la corruption et à l'exploitation des gens vulnérables. Chez Bouyoucas, le détective joue le rôle du miroir de l'humanité, le double de nous-mêmes, l'incarnation de l'aspiration à la justice, du sacrifice au bénéfice de la société, du pouvoir de la raison contre l'illusion, le préjugé et le mensonge. Le détective Soucy dans La vengeance d'un père s'inspire d'abord d'une éthique de compassion qui l'amène à promettre à Yannis Fokas de la justice. Par contre, son sentiment de devoir est empoisonné par ses propres préjugés. En se penchant sur les conflits sociaux, les divisions entre fédéralistes et souverainistes, entre immigrants et Québécois de souche, entre nantis et démunis, ce roman illustre la difficulté pour la pensée rationnelle et la justice de prévaloir contre l'abject dans une société affligée de conflits basés sur l'ethnicité, l'allégeance politique et la classe sociale. Par contre, le roman Cocorico exploite les motifs familiers du polar afin d'en faire une fable qui illustre le pouvoir de l'imagination mais aussi la course contre la mort de la faculté de raisonnement qui marque la chute de l'être humain dans l'abject. Dans le cas de La vengeance d'un père, on peut surtout parler de subversion de l'intrigue classique du polar dans la mesure où Bouyoucas refuse la solution trop facile que de fermer l'enquête par l'arrestation des malfaiteurs. Or, comme le signale Brownson, un tel refus de la clôture de l'intrigue policière signale l'écart entre le genre consacré du polar et l'œuvre plutôt littéraire qui favorise l'ouverture et l'ambiguïté (83). Ici, le polar de Bouyoucas cède la place à une analyse plus subtile des drames familiaux, communautaires et politiques. L'évacuation de l'abject en faveur de l'ordre social ne s'accomplit pas de façon satisfaisante. Dans Cocorico, le ton ludique prédomine au début mais au fur et à mesure que l'auteur Leo Basilius et son personnage Vass Levonian prolongent leur discussion philosophique, c'est plutôt l'angoisse et la démence qui risquent de noyer l'esprit rationaliste du récit policier. Ici, le détective offre ses services de collectionneur d'indices et de logicien comme rempart contre la peur de la mort et du néant, contre l'abject, en somme. Malgré le mépris de l'auteur fictionnel, les polars de Basilius, avec Levonian en vedette, constituent sa postérité. 
La figure du détective, aussi cliché qu'elle puisse paraître, offre chez Bouyoucas un idéal de devoir, de sacrifice et de logique rigoureuse menacé à tout moment par le danger omniprésent de la corruption et de l'égoïsme destructeurs.

\section{Ouvrages cités}

Blanc, Jean-Noël. Polarville ; images de la ville dans le roman policier. Lyon : PU de Lyon, 1991.

Brownson, Charles. The Figure of the Detective : A Literary History and Analysis. Caroline du Nord : McFarland, 2014.

Bouyoucas, Pan. Cocorico. Montréal : XYZ, 2011.

---. «De quelle origine est ce personnage ?». D’autres rêves; les écritures migrantes au Québec.

Dir. Anne de Vaucher Gravili. Venise : Supernova, 2000 : 97-112.

---. La vengeance d'un Père. Montréal : Les Allusifs, éd. revue et corrigée, 2016 ; Montréal : Libre Expression, 1997.

Chevalier, Jean et Alain Gheerbrant. Dictionnaire des symboles. Paris : Laffont, 1982.

Highmore, Ben. Cityscapes ; Cultural Readings in the Material and Symbolic City. Londres: Palgrave Macmillan, 2005.

Kellett, Kathleen et Kirsty Bell. «Entretien avec Pan Bouyoucas : l'écrivain, l'apprentissage, l'espace de l'écriture ». Voix plurielles 15.1 (2018) : ?

Kristeva, Julia. Pouvoirs de l'horreur. Paris : Seuil, 1980.

Mane, Robert. «Essai de topographie 'migrante' : Fenêtre sur rue dans trois romans de Pan Bouyoucas ». Stratégies du mouvement et du franchissement : la rue et le pont au Canada». Dir. Marie-Lyne Piccione et Bernadete Rigal-Cellard. Pessac : PU de Bordeaux, 2008 : 3745 .

Parizeau, Jacques. «Discours de défaite ». Archives de Radio-Canada, 30 octobre 1995. http://archives.radio-canada.ca/sports/partis_chefs_politiques/clips/14007/

Verthuy, Maïr. «Pan Bouyoucas et le principe des vases communicants ou de la nécessité de sortir de l'ethnicité ». Literary Pluralities. New York : Broadview P, 1999: 172-183.

Walsh Matthews, Stéphanie. «Le plaisir du texte : comment décoder sans dévoiler ». Semiotics (2012) : 275-283.

Xanthos, Nicolas. De l'empreinte au récit ; représentation des processus de sémiotisation de l’indice dans le roman policier. Québec : Nota bene, 2008. 